\title{
CHRONIC FOCAL INFECTIONS AND THEIR ETIOLOGIC RELATIONS TO ARTHRITIS AND NEPHRITIS*
}

FRANK BILLINGS, M.D.

CHICAGO

There is nothing new in the principle involved in the subject of the paper. It has long been known that acute rheumatic joint-infections are the result frequently of a primary infection of the faucial tonsils, or tissues about them. Pneumonia is doubtless the frequent result of the sudden change of a non-virulent to virulent type of pneumococcus whose common habitat is the upper respiratory passages in city-dwellers. It has been shown that a common source of infection in epidemic cerebrospinal meningitis is the nasal mucous surfaces. Acute endocarditis also has its source, in many instances, from the faucial tonsils. Acute parenchymatous nephritis is frequently the result of the toxemia of diphtheria. Acute gonorrheal arthritis has its source in a focal infection of the urinary or genital tract. A local tuberculous focus may cause systemic infection.

\section{SITE OF FOCAL INFECTION}

1. The faucial tonsils, the peritonsillar tissues and supratonsillar fossæ. In this may also be included the lymphoid tissue embraced in the pharyngeal tonsil and elsewhere in the nasopharyngeal space. The lymphoid tissue comprised in the tonsillar tissues is most abundant in childhood, and frequently becomes atrophic in adult life. The site of this tissue subjects it to infection of various kinds. The abundance of this tissue in the child probably explains the frequency of infections like acute rheumatic fever, diphtheria, tonsillitis, etc., in the earlier periods of life. The fact that chronic septic focal infection may lie latent in the tonsillar tissue has not been generally recognized. That this focal infection may produce a chronic systemic disease is established by clinical experimentation.

2. Abscesses of the gums and alveolar sockets, pyorrhea alveolaris and septic types of gingivitis may also cause systemic disease of various types.

3. The various sinuses about the head - maxillary, ethmoidal, sphenoidal and frontal — may also harbor focal infection and cause systemic disease.

4. Bronchiectatic and pulmonic cavities due to chronic disease may also produce chronic systemic infections.

*Read before the Chicago Medical Society, Nov. 15, 1911; received for publication Jan. 30, 1912. 
5. Chronic ulcers of the gastro-intestinal tract, especially of the bowels. This source is probably rare and more problematic than that of any other systemic infection.

6. Chronic appendicitis. Chronic catarrhal appendicitis may produce not only the local discomforts including disturbance of the functions of the digestive organs, but it may also be a focal source of systemic infection with the damage done chiefly to the cardiovascular apparatus.

7. Cholecystitis and cholangeitis, with or without calculi, have been recognized as a source of systemic infection, the brunt of the damage apparently falling on the cardiovascular apparatus and kidney.

8. The urinary tract, including the pelvis of the kidney, the bladder and more particularly the prostate gland. Pyelitis of whatever type, even when there is only moderate obstruction of the drainage of the kidney pelvis, may produce myositis, arthritis, neuritis, etc.

9. Genital tract. The prostate and seminal vesicles are a common source of infection of gonorrheal arthritis and probably of ordinary septic infections. The fallopian tubes and the uterus are less common as a source of chronic systemic disease in all probability. It is said that the parametrium is a more common focal source of infection.

10. Local, septic, submucous and subcutaneous foci anywhere in the body may be a source of systemic disease.

\section{THE SYSTEMIC RESULTS OF FOCAL INFECTION}

1. Chronic arthritis is one of the most common results.

2. Nephritis both acute and chronic.

3. Cardiovascular degenerations.

4. Chronic neuritis and myalgia (myositis).

The studies and experiments embodied in this paper are limited to the arthritides and to subacute and parenchymatous nephritis. Of these, chronic deforming arthritis, commonly known as arthritis deformans; a chronic osteoarthritis of hrpertrophic or atrophic type, is the form seen in the majority of the studies. Text to the arthritides the largest number of cases comprise subacute parenchymatous nephritis and chronic parenchymatous nephritis.

The work has been done on private and clinical patients in the Presbyterian Hospital. The bacteriological and histological studies and the animal experiments have been carried on by Dr. D. J. Davis and by Dr. Homer K. Nicoll.

\section{MIODE OF PROCEDURE}

The patient presenting eridences of chronic cardiovascular disease, nephritis or arthritis, is carefully examined in reference to previous disease which might be related to the existing condition and also for the existence of focal infections somewhere in the body. When a focal point 
of infection has been located and seems rationally related to the systemic infection, it is removed if possible, surgically or by some other means. An attempt is made thoroughly to eradicate the focus of infection. Appendectomy, drainage of the gall-bladder or of the pelvis of the kidney is insisted on not only because of local discomforts related to these diseases, but, what is quite as important, to prevent further systemic degeneration. The prostate and seminal resicles may easily be reached by the finger and the septic contents removed. If necessary, vasectomy and drainage may be carried out. Transillumination of the head may be made to determine the presence of sinusitis. The skilled specialist is necessary to remove these conditions by operation or treatment. The faucial tonsils should be enucleated. Ordinary tonsillectomy leaves an abundance of Iymphoid tissue which may be sealed over by the operative scar and leaves a worse condition than that for which the operation was made. Adhesions between the tonsils and pillars of the fauces frequently wall in foci of infection in peritonsillar tissues. The supratonsillar fossa may contain lymphoid tissue and continue a source of focal infection even when the faucial tonsil is removed. Ulcerative gingivitis, small abscesses under the gums and collections of pus in the alveoli require vigorous treatment. Ill-fitted crowns on teeth and much bridge-work may harbor septic infertion in the mouth and produce systemic disease, and when found should be removed.

Careful examination of the tissues or secretions removed by manipulation or operations have been made microscopically; cultures of bacteria attempted and, in the work we have done, animal experimentation has been carried out in many instances.

Illustrative conditions as found in patients suffering from chronic arthritis of a deforming type and subacute and chronic parenchymatous nephritis are included in this report. So far, more than thirty cases have been under close observation whose original focal infection appeared to be in the tonsillar tissues. It is impossible to give all of the results in these patients at this time because a good many of the patients are still under observation. The result of the method of treatment in the majority of the patients has been most successful. In two patients of the advanced type of chronic deforming arthritis, no beneficial result was obtained. Following the report of the patients treated, the method of treatment will be further amplified.

\section{CASE REPORTS}

\section{ARTHRITIS}

CASE 1.-Patient.-Mrs. A. P. R., aged 38, multipara, had an osteo-arthritis of left hip, which had existed for six years with shortening of the extremity and pain, stiffness and lameness on exertion with gradually increased severity. She had had four pregnancies with complicated deliveries; one miscarriage. Patient was well nourished and presented, in addition to the disease of the left hip, hypertrophied and infected faucial tonsils, ruptured perineum with a retro-displaced 
large uterus. Blood: hemoglobin 80 per cent., reds $4,350,000$, whites 8,000 . Twenty-four-hour collection of urine, 1,525 c.c., specific gravity 1.010 , acid, no, albumin, no sugar, no casts, no red cells, few lenkocytes. $X$-ray of hip showed' erosion of upper border of acetabulum with mushroom enlargement at juncture of head and neck of the femur, some flattening of the head.

Treatment and Animal Experiment.-Plastic pelvic operation and uterine curettage was done by Prof. J. C. Webster. The tonsils were thoroughly enucleated by Dr. George E. Shambaugh. From the cut surface of the tonsils a nearly pure streptococcus was obtained in culture. A rabbit was inoculated with resulting acute multiple arthritis. In a few days the animal died, and from the infected joints and the heart's blood of the animal pure cultures of the streptococcus were obtained.

Course.-The patient was given a modified rest cure. Six months after leaving the hospital a letter from the patient stated that she was feeling well, could walk, play golf, ride horseback and perform any physical effort without discomfort. A slight limp due to shortening of the left extremity remained.

CASE 2.-Patient.-Mrs. J. W. J., aged 40, quartipara, presenting a case of osteo-arthritis atrophica chronica, was first seen in January, 1907. For about eighteen months the patient had had swelling and stiffness and pain on motion with some deformity of the small joints of hands, feet, knees, wrists and elbows and more recently stiffness and lameness on moving, in the cervical and dorsal spine. Previous condition of health was good. During the whole period of illness she had had treatment for chronic arthritis in which salicylate of soda and other antirheumatic remedies were used. Recently a "streptolytic" serum was given by deep injection, as much as 100 c.c. per day for four days and then repeated on the sixth. Reaction with temperature of $102 \mathrm{~F}$. and general malaise followed each injection. 'The patient was weak, nervous, with poor appetite and general stiffness on exertion in the affected joints. Menstruation was regular.

Examination.-Blood, hemoglobin 50 to 55 per cent., reds 4,100,000, whites 12,500. Urine: twenty-four-hour collection normal. Tonsils moderately enlarged, adherent to the pillars of the fauces and crypts moderately infected. General examination as to the organs of the body was negative. Pelvic organs normal. Many of the joints of the fingers were spindle-shaped, erepitated on motion; both wrists were enlarged and fluctuated. Swelling and tenderness of small joints of feet and more especially of the metatarsophalangeal, cervical spine, tenderness on motion. Rest cure treatment and summer spent at Asheville, N. C., gave no relief. There was progressive development of the disease in all of the joints.

Treatment.-Aug. 15, 1907, the tonsils were thoroughly enucleated by Dr. Otto T. Freer. A rest treatment was then instituted with restorative tonics. Treatment with syrup of iodid of iron immediately.

Course-Following the tonsillectomy all of the joints became considerably worse and there was redness, swelling and pain on motion. The patient could walk only with great discomfort. In April, 1908, there was a great improvement. The patient was able to walk $21 / 2$ miles a day without fatigue or pain. There was still some deformity of the joints of fingers and some tenderness along the course of the right musculospiral nerve. Blood examination showed normal condition. In 1910 patient was reexamined and normal conditions found. The patient expressed herself as feeling entirely well. No cultures were made from this patient's tonsillar tissues.

CASE 3.-Patient.-Mrs. E. W., aged 50, primipara, with a osteo-arthritis chronica of mixed type, was admitted to the Presbyterian Hospital Oct. 16, 1909. For two years there had been swelling, tenderness, pain on motion and deformity of many of the joints of extremities. It began in feet and hands and extended to larger joints and finally involved cervical spine. The condition was progressive. There was malnutrition, loss of weight from 160 to 129 pounds. For years the 
patient had been subject to attacks of acute tonsillitis. She had also suffered for years from pyorrhea alveolaris.

Examination.-Patient was poorly nourished, very nervous and irritable. Her mouth was badly infected, with many stumps of carious teeth, some of them loose in the sockets, gums retracted and infected, tonsils large, rough, adherent to pillars of fauces and crypts infected. Her breath was offensive. Heart, lungs, abdominal organs and pelvic organs were normal. There was swelling with some deformity of both ankles, right metatarsophalangeal, both knees, right middle and left fingers, the wrists and elbows. Some contraction of hamstring muscles prevented complete extension of legs. Both biceps tendons of arms contracted which prevented extension of forearm. Twenty-four hour collection of urine was normal in amount and specific gravity and contained a few hyaline casts. Blood: hemoglobin 90 per cent., reds 4,600,000, whites 13,400 . Oct. 18, 1909, both faucial tonsils were enucleated by Dr. George E. Shambaugh and one week later the roots of carious teeth were thoroughly removed by Dr. Frederick Moorhead. From the cut surface of the tonsillar tissue a pure culture "of streptococcus was obtained.

Animal Experiment.-A rabbit inoculated with a culture suffered from acute multiple arthritis and died in a few days. The streptococcus was regained from the infected joints and from the heart's blood.

Course.-The patient was permitted to return to her home too soon and did not fully carry out directions as to rest treatment. Some time elapsed before the alveolar processes were absorbed and the mouth remained sore. April 3, 1910, she returned to the hospital where rest treatment was instituted with resulting marked improvement. The patient gained in weight from 129 to 140 pounds. After the return home frequent communications by letter with the patient and her physician have shown that the progress of the disease has entirely stopped. Some of the deformities were so great that one could not expect entire anatomical restoration. The last communication is dated December, 1911, in which the patient says that the strength of her upper extremities and spine is entirely normal. There is some fatigue in the lower extremities after attempting to walk for any great distance, but there is a continued improvement even in this respeci.

CAse 4.-Patient.-J. H., male, married, aged 37, bookkeeper, with osteoarthritis hypertrophica chronica, was admitted to the Presbyterian Hospital Dee. 9, 1909. There had been swelling, tenderness and soreness in the knees, ankles, left wrist and right jaw and right temporomaxillary joints, during the past four rears. The first trouble began in the right knee following a fall. A diagnosis of tuberculous arthritis was made at that time and the knee-joint was injected with iodoform emulsion on four different occasions. This gave no relief. After one year the left knee became involved and this was also aspirated. Then followed the involvement of other joints named. Recently the patient has had tonsillitis, but does not recall that he suffered from the disease since childhood. He denies venereal disease, has four healthy children, and is well except for the arthritis.

Examination.-Both tonsils were greatly enlarged, rough and many follicles showed cheesy masses. Patient is unable to fully open the mouth. Both knees greatly enlarged, the right measuring 40 , the left $39 \mathrm{~cm}$. Motion limited. Small rice-like bodies can be felt in both joints. Both ankles are swollen, tender, slightly limited in motion. Spinal column is not involved. General examination did not show any abnormal find of importance. Blood: hemoglobin 72 per cent. (Dare), reds 4,750,000, whites 9,500. Prostate not enlarged; no exudate obtained from stripping prostate. December, 1909, both tonsils were thoroughly enucleated by Dr. F. Guerney Stubbs. Cultures from cut surfaces of tonsils yielded hemolytic streptococcus in pure culture. A rabbit inoculated with the streptococcus developed acute multiple arthritis and died in one week. A streptococcus was obtained in pure culture from the joint exudate. The heart's blood was sterile. Fluid from the right knee to the amount of two ounces was cloudy, yellow and 
sterile. Patient left the hospital December 18, improved both as to joint condition and general health. A report recently from him stated that he had practically returned to normal condition.

CASE 5.-Patient.-Dr. A. W. A., aged 40, married, with osteo-arthritis hypertrophica chronica of spine, was admitted to the Presbyterian Hospital March 20, 1911. Complained of stiffness and soreness with pain on motion in hips and shoulders which began five years before as an attack of apparent lumbago. The condition gradually involved the whole back and during the previous winter pain had extended to the left side of the neck and radiated around the chest, making it difficult to breathe. Stiffness and soreness increased after lying or sitting, aggravated by jars in walking or driving. Periods of improvement followed by worse conditions. At one time the joints of the fingers were involved during acute exacerbation of the spinal trouble. He did not remember that he ever suffered from severe tonsillitis. Had lost 30 pounds in the previous year. Had a gonorrhea of mild type fifteen years ago.

Examination.-Tonsils were small, adherent to pillars of fauces, and some of the crypts could be seen infected. Heart and lungs were normal. Abdomen was negative excepting for rigidity of abdominal wall. Spinal column was rigid. There was tenderness to pressure along the spinous process; some tenderness on motion of both shoulders and hip-joints but no crepitation. Prostate was not enlarged, not tender and no exudate was obtained by stripping the glands. March 25, 1911, both tonsils were thoroughly enueleated by Dr. Feo. E. Shambaugh. Tonsillar tissue small in amount, but small abscesses were found deep in the tissues from which a pure culture of streptococcus was obtained.

Animal Experiment.-A rabbit inoculated intravenously developed acute arthritis which progressed into a chronic form of osteo-arthritis. The animal lived nearly two months, became emaciated and at the time of death had several enlarged joints with exostosis and subluxation of the left shoulder. A streptococeus was isolated from the exudate in the joints.

Course.-The patient steadily improved, played golf during the summer and followed his professional duties. A letter dated Dec. 18, 1911, states: "My general condition is extremely good. I have added 30 pounds to my weight and have lost the sense of fatigue from which I suffered. I can move much more rapidly and comfortably and rest well at night. I still suffer from periods of decline, but these are very short in duration. My spine has improved wonderfully, I can stand almost straight and the pain is almost gone. There is still some limitation in motion especially in the neck, but there is steady improvement."

CASE 6.-Patient.-Dr. E. S. E., aged 26, single, with polyarthritis subacuta, was admitted to the Presbyterian Hospital March 2, 1911. Pain in both knees, left ankle, small joints of both feet, especially of the metatarsophalangeal, and some soreness in lumbar spine. Tonsillitis began ten years ago and, after that time an exacerbation of an arthritis of feet and knees was exaggerated. Was obliged to go to bed. Had an attack of tonsillitis with arthritis six years ago.

Examination.-There was swelling and tenderness of joints named. The tonsils were large, rough, crypts infected with whitish exudate. Lungs and heart were normal; abdominal organs normal.

After two weeks of treatment the patient was discharged as subjectively well. Iater the patient developed chronic arthritis involving small joints of feet, ankles, linees and wrists. In January, 1911, he had acute appendicitis which was operated on. Before the patient left the hospital his tonsils were enucleated by Dr. Friedburg.

Animal Experiment.-A pure streptococcus was obtained in culture and inoculated into a rabbit. Acute multiple arthritis developed and the rabbit continued ill for a considerable period and then recovered.

Course.-Following the enucleation of the tonsils the patient passed two weeks in the country at rest. Improvement was steady and progressive and by the 
middle of September, 1911, he was entirely free from all joint involvements and able to return to his professional work.

CASE 7.-Patient.-Mrs. C. C. M., aged 30, primipara, with osteo-arthritis atrophica chronica, was admitted to the Presbyterian Hospital May 17, 1911. Patient complained of soreness, stiffness and swelling and some redness in the small joints of feet and hands, knees, shoulders and wrists. The joint-trouble appeared first in September, 1910, and continued until February, 1911, when there was a rather sharp exacerbation. The patient was always worse in the morning; had had no fever. She was nervous, had a good deal of occipital headache and pain in the back. Appetite and digestion were good; bowels moderately constipated. Average weight was 108 pounds. There was no disturbance of the urinary organs. Menstruation regular, lasting four days, but painful. Had a miscarriage three and a half years ago. Had frequent sore throat since an attack of scarlet fever when she was a child. Pain in left side few years ago, which was called "pleurisy."

Examination.-Patient was frail and sallow; tonsils enlarged, rough, adherent to pillars and yellowish exudate could be seen in crypts. Lungs and heart were normal. Abdominal organs appeared normal. Pelvic examination showed no abnormal condition. Urine, twenty-four hour collection, was normal. Blood: hemoglobin 75 per cent., reds 4,600,000, whites 6,000. March 23, 1911, tonsils were thoroughly enucleated by Dr. George E. Shambaugh.

Animal Experiment.-A pure streptococcus was obtained in culture from the cut surfaces. This inoculated into a rabbit produced an acute multiple arthritis. The animal died and the culture of streptococcus was obtained from the exudate of the joints.

Course.-Twenty-four hours after the enucleation of the tonsils, patient left the hospital without my knowledge. She kept up and about and suffered more with joint-pain than for a long time. She was then put to bed on a rest-cure treatment and immediate improvement occurred. Sept. 25, 1911, patient reported herself as improved in every way. Joint-trouble was practically gone. She still complains of lessened strength and endurance.

CASE 8.-Patient.-Miss M. I. P., aged 39, nurse, with arthritis atrophica chronica, was seen first Dec. 7, 1910. Patient complained of swelling and stiffness in both knees which began a year and a half ago; constantly present, but periodically worse. There was no pain when patient was quiet; it was aggravated by motion and fatigue. Left index-finger became involved in 1910 and later the right elbow. Tonsillitis of and on for four years. Had noticed an increase of joint-disturbance with tonsillitis. General condition was good. Bowels regular; menstruation normal.

Examination.-Patient well nourished. Teeth good; mouth clean, tonsils enlarged, rough, both infected, right more than left. Heart and lungs normal; abdominal organs negative. Blood: hemoglobin 85 per cent., reds 4,600,000, whites 9,000. Urine, twenty-four hour collection, showed normal condition. Both kneejoints were swollen, crepitated on motion and were tender. No exudate. Right elbow swollen and tender. Left index-finger spindle-form. Tonsils enucleated by Dr. George E. Shambaugh.

Animal Experiment.-Cultures made from cut surfaces of tonsils gave a streptococcus in almost pure culture. Streptococcus injected into rabbit produced acute multiple arthritis. The animal died and from the exudate of the joints and the heart's blood a pure culture of the streptococcus was obtained.

Course.-June 27, 1910, patient wrote that she had taken a rest-cure for ten days and had resumed her work in a graduated way. There had been slight but gradual improvement until about middle of May; then she rapidly improved and considered herself entirely well.

CASE 9.-Patient.-Mr. I. M., aged 27, single, traveling salesman, with osteoarthritis hypertrophica chronica, was admitted to the Presbyterian Hospital May 25,1911 . He complained of limitation of motion and pain in right elbow, both 
knees, left sternoclavicular and left temporomaxillary. Trouble began three years ago; onset insidious. It began in knees with swelling, pain on motion. Two months later toes, fingers, elbows, shoulders and jaw became involved. Patient was treated at hospital in Pittsfield, Mass., and cast put on one knee. This gave temporary relief. Since that time the course had been one of exacerbation and decline, but the patient had never been free from some debility. He had had scarlet fever with double otitis media when a child. He denied venereal disease, did not remember to have suffered from tonsillitis.

Examination.-Right forearm could not be fully extended because of contraction of biceps muscle. Elbow-joint was not involved. Left knee-joint was swollen with effusion into synovial sac. Soft structures about joint were boggy and thickened; hamstring muscles contracted. Right knee-joint showed abnormally free motion. There was exostosis on external surfaces of outer condyle. Left sternoclavicular articulation was moderately swollen, tender and painful on motion and mouth could not be fully opened. Other joints were normal. Lungs and heart were normal. Mouth was clean; teeth sound. Tonsils were not enlarged, but were ragged and buried between adherent pillars, showing point of exudate in follicles. Abdominal organs were normal. Prostate was not enlarged and no exudate could be obtained upon stripping the organ. The urine in single and twenty-four-hour collection was normal. Blood: hemoglobin 76 per cent. (Dare), reds $4,100,000$, whites 8,400 . The tonsils were thoroughly enucleated by Dr. George E. Shambaugh. Thirty c.c. of a clear fluid was obtained from the left knee-joint. This was sterile and microscopically only a few polvnuclear leukocytes were shown.

Animal Experiment.-A streptococcus in pure culture was obtained from the cut surfaces of the tonsils. A rabbit inoculated developed acute multiple arthritis and died in a few days. A pure culture of streptococcus was obtained from exudate of joints.

Course.-Autogenous vaccinations were made and used on this patient every week or ten days. June 25, he showed marked improvement and in September he returned to his usual occupation which he followed without much discomfort. December 22 the patient wrote that his condition was much improved and that there was practically no pain in the joints and very little if any stiffness. Slight swelling still remains in the left knee. He had had three vaccinations and to the vaccinations he ascribed much of his improvement.

CASE 10.-Patient.-W. J. F., aged 38, married, brewer, with arthritis rheumatica chronica, was admitted to the Presbyterian Hospital Sept. 11, 1911. He had suffered from attacks of rheumatism, beginning when he was 17 years old, with frequent attacks during the last twenty-one years. Finally chronic arthritis resulted. There was a decided rheumatic family history. Patient had gonorrhea fifteen years ago from which he made a good recovery. On admission to the Presbyterian Hospital he presented swollen, painful right ankle, right index and second fingers and right elbow. Tonsils were enlarged, rough and infected. The left border of the heart measures $12 \mathrm{~cm}$. from the midsternum. A soft systolic murmur was heard in right second interspace, not transmitted. Urine examination normaI, including specimen and twenty-four-hour collections. Blood-examination, normal find. Prostate not enlarged and exudate could be stripped from the gland. September 26 the tonsils were thoroughly enucleated by Dr. George E. Shambaugh.

Animal Experiment.-From the cut surfaces of the tonsils almost pure cultures of the streptococcus were obtained. This inoculated into a rabbit produced acute multiple arthritis from which the animal died in a few days. The streptococcus was reobtained from the joint exudate and heart's blood.

Course.-Phenomenal improvement followed the enucleation of the tonsils. The first two or three days the joints were more swollen and painful. Then immediately began improvement and for the first time in many years the patient is now entirely free of arthritis. 
These case histories are good examples of arthritis deformans and include but a few of the many who have been so managed, but are sufficient in number to illustrate the principles of the paper.

SUBACUTE AND CHRONIC PARENCHYMATOUS NEPHRITIS

CASE 1.-Patient.-Miss L. R., aged 22, of Chicago, was admitted to the Presbyterian Hospital Sept. 11, 1909. Three or four years previously she had had an attack of acute tonsillitis with bronchopneumonia. Following this, edema of face and hands developed a pasty pallor, headache and nausea, and it was found that the urine contained albumin and a good many hyaline, granular and epithelial casts.

Examination.-Marked pallor, edema of face, lower extremities with some free fluid in the abdominal cavity. Heart and lungs negative. Liver not enlarged. Spleen not palpable. Blood: 4,000,000 reds, 18,700 whites, 63 per cent. hemoglobin (Dare). Urine: twenty-four-hour collection 900 c.c., specific gravity 1.020, much albumin, many hyaline and granular casts, much blood. Arterial tension (Stanton), $145 \mathrm{~mm}$. systolic. Tonsils very large, rough, infected, with adhesions to the pillars of the fauces.

Treatment.-The patient was kept under treatment at the Presbyterian Hospital from September 11 to November 13, on a suitable diet, free bowel actions every day and an iron alkaline treatment with gradual improvement in general condition with a better urine output, less general dropsy, with an improvement in the blood condition, but on the whole the patient did not advance much toward recovery. Therefore, Jan. 5, 1910, she was again placed in the Presbyterian Hospital and the tonsils were thoroughly enucleated by Dr. F. Guerney Stubbs. The patient made an uneventful recovery from the tonsillectomy. The patient was kept quiet on a suitable diet, restorative tonics, etc. January 19 the twentyfour-hour collection of urine was 1,470 c.c., specific gravity 1.015 , albumin 0.08 per cent. Three fine granular casts were found in the centrifuged specimen, no blood, no leukocytes. Before this date the albumin was larger in quantity. There were always many casts and a good many red cells and leukocytes. From this date on the urine slowly cleared up.

Course.-The general condition of the patient improved until the winter of 1910 and 1911. When in the south (San Antonio, Tex.) she acquired bronchopneumonia and was severely ill for a time. Nevertheless recovery from this condition followed with a continued improvement of the kidney and general condition. June 24, 1911, a morning specimen of urine showed no serum albumin, no casts, no blood, no leukocytes. An evening specimen, specific gravity 1.024, showed no albumin, no casts, no blood. The blood at this time showed hemoglobin 90 per cent., reds 5,400,000, whites 8,000 . General hygiene was continued and at the present time the patient is practically well. October 17,1911 , there were 1,400 c.c. of urine, specific gravity 1.012, no albumin, and the sediment from a fresh specimen examined at the same time showed no leukocytes, no blood-cells, no casts.

Case 2.-Patient.-J. H., schoolboy, aged 13, was admitted to the Presbyterian Hospital from the Central Free Dispensary on Dec. 4, 1909, suffering from subacute parenchymatous nephritis of the hemorrhagic type. He was operated on for hernia at the Presbyterian Hospital in September, 1909, and left the hospital well. He suffered from tonsillitis three weeks previous to second admission for which no treatment was given. One week previously he vomited in the morning after breakfast, again at dinner and supper time. Frequent urination occurred at the same time and it was noticed that the urine was red in color. He became thirsty, with weakness, dizziness and two days ago he vomited up some bright red blood. Since then he had been in bed. He had scarlet fever two years ago; no other disease excepting hernia for which he was operated on, as mentioned. He had had severe attacks of acute tonsillitis.

Examination.-Poorly nourished boy, marked pallor, edema of eyes, face and some of the lower extremities. Heart and lungs negative. Abdomen negative. 
Blood-pressure in recumbent posture 135 to $145 \mathrm{~mm}$. systolic. Tonsils large, rough and infected. Temperature slightly elevated but subfebrile. Pulse and temperature practically normal. Blood: reds $3,250,000$, whites 9,400 , hemoglobin 50 per cent (Dare). Urine: twenty-four-hour collection 1,200 e.c., specific gravity 1.008, cloudy, red with blood, acid reaction. Albumin 0.1 per cent. by weight. Many twenty-four-hour collections of urine were made with practically same results. Tonsillectomy was advised and enucleation of tonsils was done by Dr. F. Guerney Stubbs January 17 . The boy made an uneventful recovery from the operation. He was kept quiet in bed on a suitable non-nitrogenous, non-salt diet; the bowels were free and as soon as the hemorrhage in the urine ceased, he was given restorative iron tonics. The blood improved gradually. January 10, a little more than a month after admission, the reds were 4,500,000, the hemoglobin about 70 per cent. Urine, the same date, 1,500 c.c., specific gravity, 1.010, acid, albumin present in small quantity, no blood, no easts, a few leukocytes. Subsequent examination of the boy during the summer of 1910 showed restoration to the normal.

CASE 3.-Patient.-Dr. R. N. S., aged 26, of Chicago, was first seen on Dee. 13, 1909, and was admitted to the Presbyterian Hospital on Dec. 15, 1909. Patient came because albumin had been detected in the urine in October, 1909. As high as 2 per cent. by measurement had been found. A few hyaline and finely granular casts had also been detected. Patient tired easily, had some dull headache and was worried much about kidney condition. He had suffered from attacks of tonsillitis for years past; had one attack during last winter. He had smoked to excess until recently.

Examination.-Tonsils large, red, ragged, infected. Otherwise throat and mouth normal. Chest, heart and lungs negative. Abdomen negative. No edema of lower extremities. Urine: specimen showed specific gravity 1.024, a good deal of albumin, not measured, many hyaline and granular casts, no blood. The arterial tension with the Stanton instrument was $95 \mathrm{~mm}$. systolic. Blood: Jemoglobin 85 to 90 per cent., reds $4,500,000$, whites 8,600 .

Treatment and Course.-Dec. 16, 1909, the tonsils were thoroughly enucleated by Dr. Rhodes. Since that time the patient has been examined and the urine is found entirely clear, and the patient remained in good health. A letter from the patient, dated Dec. 20,1911, states that the urine is free of albumin and casts. "Am feeling well and have had no evidence of nephritis for the last year and a half."

Case 4.-Patient.-R. Y., aged 18, schoolboy, was first examined May 13, 1910. A year and a half preceding this time a physician had found albumin and many leukocytes in the urine. There had been no headache, no edema and, the patient thinks, no fever. He had suffered from scarlet fever, pneumonia twice, the last time one year previous to examination and had had many attacks of tonsillitis.

Examination.-Well-developed, well-nourished boy of fairly good color. The lymph-nodes in posterior triangles of neck moderately enlarged. Tonsils enormously enlarged, red, ragged, and crypts filled with yellowish exudate. Heart and lungs both normal. Abdomen negative. No edema of face or legs. A specimen of urine showed specific gravity of 1.012 , albumin in rather large amount and the microscope showed hyaline and granular casts, and a few kidney epithelia and leukocytes. The arterial tension 130 systolic.

Treatment and Course.-The important relation of the kidney to the infection of the tonsils was explained to the parents and the attending physician, Dr. W. S. Picotte of Ishpeming, Mich., and thorough enucleation of the tonsils advised. This was done, but I fear inadequately, during the summer of 1910 . The patient spent the next winter in Florida in school and reported that his general condition had been much improved. On Sept. 12, 1911, he came for examination. The bases of both tonsils still remained and were red, somewhat infected, the general condition good, the urine acid, a faint trace of serum albumin, but no casts and no leukocytes. Arterial tension 125. The condition was explained as improved 
and regret expressed that the tonsils had not been really enucleated. Patient was advised to have urine reexamined from time to time and if albuminuria continued to have remaining portions of tonsils dissected out.

Case 5.-Patient.-Miss S. 'T., aged 20, was first examined through the courtesy of her physician, Dr. Albert Smith of Parsons, Kansas, July 6, 1911. The complaint was extreme weakness, swelling of the lower extremities, the abdomen and anemia. The history showed that in November, 1910, the patient had a cold with infection of the throat. About a month later patient became very tired at a dance and it was noticed that the feet became swollen. Albumin and casts with blood were found in the urine. The patient was taken to Texas at some mineral springs and drank quantities of water. Rapid edema of face, extremities and body resulted. She had had no headache, no fever and no disturbance of vision; slight cough recently. The patient had scarlet fever at 7 years of age.

Examination.-A tall slim girl, very pale, marked edema of face, lower extremities, free fluid in peritoneal and a moderate amount in both pleural cavities. Few lymph-nodes palpable in posterior triangles of neck. Apex of heart $8 \mathrm{~cm}$. from midsternum. A blowing murmur over the pulmonary area. Arterial tension $140 \mathrm{~mm}$. systolic, pulse 78 per minute, temperature normal. Blood: hemoglobin 53 per cent. (Dare). Urine: specific gravity 1.012, much albumin, many hyaline and granular casts and many red cells and leukocytes. A good many kidney epithelia. The patient was placed in Presbyterian Hospital on an appropriate salt-free diet. At the hospital the urine measured in twenty-four hours from 600 to 650 c.c. on different days, specific gravity 1.018 to 1.020 , contained a great deal of albumin, many hyaline and granular casts, many leukocytes and kidney epithelia and a good deal of blood.

Treatment and Course.-The tonsils were enucleated by Dr. Friedburg and the weakness of the patient necessitated two operations with an interval of one week between. The dropsical condition gradually diminished and the patient improved in general condition. July 21 there were 1,620 c.c. of urine, specific gravity 1.010, with less albumin, fewer casts and no blood. July 29 the total urine was 1,620 c.c., specific gravity 1.010 , little serum albumin, few hyaline and granular casts, few red cells and few leukocytes and kidney epithelia. At this date the patient left the hospital and spent the remainder of the summer in Canada following out a rest treatment with non-nitrogenous salt-free diet and a restorative tonic of the syrup of the iodid of iron, free bowel actions daily. Sept. 29,1911 , a reexamination showed a much improved condition. There was but a trace of albumin in the urine, very few hyaline casts; the dropsy had entirely disappeared except that when the patient was up and about for the greater part of the day, there was a slight swelling about the ankles. The red cells were 4,800,000 , hemoglobin 65 to 70 per cent., whites 10,000 . A letter recently from the patient's mother tells of still further improved condition. Dec. 23, 1911, the urine examined showed specific gravity 1.015, yellowish-brown color, small amount of serum albumin and centrifuged deposit showed few leukocytes and two hyalogranular casts. Jan. 4, 1912, a letter from her physician, Dr. Albert Smith, states that the physical examination showed a slight edema above each ankle. Patient looked well; had gained in weight and strength. Blood-count at that date showed 4,252,000 reds, 8,200 whites, 80 per cent. hemoglobin, and a letter from the mother of same date states that the patient is feeling fine, does not seem to have any aches or pains and is gaining in flesh and strength.

CASE 6.-Patient.-Mr. C. L. P., aged 23, foreman in wholesale tailoring establishment, Chicago, was examined May 6, 1911. He came for examination because albumin and blood had been found in urine. Patient was easily exhausted after exertion and also early in the morning. He slept well as a rule. Appetite was not disturbed. Bowels moved regularly. Patient had had varicose veins of legs for a long time; had suffered from acute tonsillitis on several occasions during the last few winters. He had typhoid at 12 years of age; no other serious illness.

Examination.-Moderate pallor of skin, no enlargement of lymph-nodes. Tonsils both very large and infected. Heart apex $9 \mathrm{~cm}$. from midsternum, left 
border $11 \mathrm{~cm}$. No irregularity. First sound moderately rough and impure. No accentuation of second sounds. Lungs normal. Abdominal organs negative. No edema of lower extremilies. Urine showed a great deal of albumin, many casts, red celis, leukocytes and kidney epithelia. Blood: hemoglobin 70 per cent., reds $4,000,000$, whites 8,000 . Arterial tension $110 \mathrm{~mm}$. systolic. Condition of kidneys and probable relation to local infection of tonsils explained to the patient, and he was advised to have the tonsils thoroughly enucleated.

Treatment and Course.- - Yov. 4, 1911, the patient was seen by request. He had had the tonsils thoroughly enucleated by his own physician and had had a month's vacation which he spent in the country on simple non-nitrogenous food and had kept the bowels moving freely every day. At this time a specimen of urine contained no albumin, no casts, no red cells and only a few white cells. The patient expressed himself as feeling entirely well. Nov. 4, 1911, a specimen of urine examined, showed no serum albumin, no casts, a few leukocytes, no reds.

Reports of other cases are not made at this time because it would make an article of this kind too long, but subsequent reports will be made on other patients.

THE RFFECT OF TIE IXOCULATION OF ANIMALS WITH THE CULTURES OBTAINED FROM INFECTED TISSUES

It is noteworthy that the streptococcus obtained in almost pure culture from many of the patients, when inoculated into animals, produced an acute arthritis either single or multiple, and in many of the animals produced an arthritis of deforming type. Furthermore, from the dead animals' tissues the streptococcus has been again obtained in cultures. It is also true that the cultures from the tonsils of patients who had no eridence of systemic infection contained the streptococcus practically identical with those patients who had sustemic disease. Inoculation of the strains of the streptococci obtained from the tonsils of individuals, without systemic disease, has not yet been experimented with enough to know what the result on animals would be. It is an experimental fact that the streptococcus of any source may produce acute joint-lesions in inoculated animals, especially rabbits. In most of the animals inoculated with the strain of streptococcus obtained from our patients, the usual acute arthritis occurred. In some of the animals the joint-process became shronic and developed the marked anatomical changes (exostoses, rice bodies, atrophy of cartilage, subluxation, etc.) found in osteo-arthritis of man. It is also notable that in three of the patients suffering from nephritis, the strain of the streptococcus obtained from the tonsillar tissues of these patients produced albuminuria in the inoculated animals. No other strains produced albuminuria.

A few of the patients have been treated with autogenous vaccines. The vaccines invariably produced reactions when injected in the dose of from 10 to 500 millions. This reaction consisted of marked tenderness, redness and swelling and frequently of rise of temperature of varying degree with constitutional disturbance in the form of general discomfort, aching muscles, headache, etc. Leukocytosis of varying degree would sometimes follow the injections. Tenderness in joints would also sometimes occur 
with the vaccinations. So far it cannot be said that the autogenous vaccinations have been of any especial value in a group of patients. Individual patients have apparently shown greater improvement with the vaccinations than without them.

General hygienic treatment has been necessary in all the classes of patients treated and one would naturally infer that this general treatment had a good deal to do with the result. With the arthritis cases it has been found just as necessary to continue a long and yet variable rest treatment with good food, restorative tonics and the various forms of individual treatment usually employed in the management of this group of diseases. Assistance has been rendered by Dr. John Ridlon, Dr. Charles A. Parker, Dr. E. W. Ryerson, and others in the arthritis cases, by such measures as were found necessary in individual patients. Casts have been applied to extremities, or to the spine, contracted tendons have been stretched and other necessary manipulations and corrective measures used.

In those patients who, for some reason, could not or would not follow out the details of after-treatment, rest, etc., improvement was not as soon obtained or not as fully secured as in those patients under command. This, however, does not mean in my opinion that the final results are due to the after-treatment alone, for in past years the same care in the general treatment did not give the same degree of good results that has been obtained in later years by the methods suggested in this paper.

\section{FURTHER EXPERIMENTATION NECESSARY}

It naturally occurs to one that there must be some other element present in individuals or something lacking in some patients which permits the systemic disease to attack them while others escape who present practically the same local condition with chronic focal infection. Many people suffer from chronic hypertrophic tonsillitis with chronic infection, or an excess of lymphoid tissue in the nasopharynx with consequent anatomical. obstruction and lack of drainage, or have chronic sinusitis, or chronic focal disease in other portions of the body and yet appear to be entirely well. Naturally the hypothesis suggests itself that there must be some protective agency present in certain people and lacking in others. It is a well-known fact that traumatism, excessive fatigue, general debility from some previous exhausting disease or exposure may be the beginning of an arthritis. We commonly speak of this as a less resisting power in the individual. Can we by some means ascertain what the essential lacking element may be in the individual whose joints are attacked from an existing focal infection? The specificity of the bacteria obtained by cultures from focal infections could probably be proved by investigation of the reaction to blood-serum, the spinal fluid and the exudate in the joints or elsewhere of patients suffering with systemic disease and some light could probably be thrown on the question by the research into the question of bacterial reaction to the blood, spinal fluid, urine, etc., of 
patients who hare focal infection without systemic disease. A further study would be of value also of the opsonic index and other specific reaction of the blood, spinal fluid, joint fluid, ete. of individuals before and after autogenous vaccinations or after inoculations of polyvalent horseserum made by injection into the horse of several strains of streptococci obtained from patients. Research of this kind is under way in the work which is now being done and it is to be hoped that a later report may explain some of the conditions which are now obscure.

I think that there can be no doubt that the insidious, slow, degenerative processes which occur in many patients who arrive at the meridian of life are due to slow intoxications from chronic focal. infections variously located. The medical literature is full of statements of auto-intoxications from the intestinal tract and it is not improbable that this may be a source. It is a fact that with proper hygienic management in diet, exercise, free excretion through the bowels, kidney, skin, etc., retrograde changes are delayed and an individual's health much improved thereby, but it is also true that with these measures fully carried out many people still show progressive interstitial nephritis with cardiovascular changes in spite of the best hygienic management. Not infrequently it has been my experience to find that such patients suffer from a chronic infection of the gall-bladder, appendix or prostate, or some other focal infection. The result of the removal of these infections has also been most astounding in many instances. This paper would be too long to discuss further that part of the result of focal infections. I hope to present that phase of the subject in another paper in the near future.

TIIE NECESSITY FOR THE REMOVAL OF FOCAL INFECTIVE PROCESSES

It is not the purpose of this paper to adrocate unnecessary surgical procedure. It is, however, necessary to do something radical when one finds a patient who suffers from a focal disease that may produce acute disease or chronic degenerative changes in any of the organs of the body. Excessive amounts of lymphoid tissue of the faucial tonsils or nasopharynx in children is a double menace. With obstruction and want of drainage those localities produce not only alterations in the bony framework of face and chest, but offer a source of systemic infection which may be ruinous to the individual. There can be no other reason for the prevalence of rheumatic fever in children than the frequency of local infections in throat and nose. Quite as frequently children have endocarditis without other symptoms or rheumatic infection which has its source in the throat. There may possibly be a reason for the retention of some of the lymphoid tissue in the faucial tonsil, but so far, the physiologists have not been able to give a reason for its retention. Nor, I think, has there ever been an argument advanced to show harm from 
the practical enucleation of all of this tissue. I think anyone who has noted the difference in the physical and mental makeup of a child before and after the removal of large amounts of lymphoid tissue in the throat must be convinced that the change is due not only to a better air-space, but also probably to the removal of a constant, even though it may be slight, systemic infection. It is an interesting observation of Dr. Thomas L. Gilmer and of others, of foci of specific infection about the gums and the presence of abscesses about the roots of the teeth, often unsuspected which must be recognized as a source of systemic infection that has not received the attention from the medical profession which it deserves. I think that any one who has seen the illuminating results of a better physical health in patients whose dirty mouths have been made as nearly as possible clean, must be convinced of this source of systemic infection.

I think that the specialist as well as the general practitioner has not fully recognized the importance, not only of the relation of the focal infection to systemic disease, and have not been fully awake to the necessity for entire eradication of the focus. Partial removal of the faucial tonsils by whatever means frequently leaves a condition as bad as the original one, or worse. An infected tonsil or tonsillar tissue need not be large in bulk. In some of our patients small and apparently innocent tonsils contained abscesses from which pure cultures of streptococci were obtained. The scar of operation may seal over infected crypts in lymphoid tissue. An incomplete operation on the mouth may still leave alveolar abscesses. In several instances of patients included in this series, more than one operation on the tonsils have been made necessary because of incomplete removal of the focus of infection by the first operator.

The purpose of this paper has been to call the attention of the profession to a source of general disease which, while recognized by a few, is entirely ignored by the many. No one would have the effrontery to say that the lack of recognition of the focus of infection is absolutely detrimental to the best interest of the patient; nor would one say that the treatment advocated is specific in that all patients are made whole and well by it; but it is believed that there is a principle involved as a cause of systemic disease which should be recognized, should be sought for more frequently and when the focal infection, wheresoever it may be located, seems to be related to the systemic disease, radical measures should be instituted to remore it. When this is done, those general measures which long have been recognized as essential to the well-being of an individual should be instituted so that nothing will be left undone whichl may restore an invalid to health. So far the experimental work done seems to prove clinically the truth of the principle advocated.

122 South Michigan Boulerard. 